Recovering an Optimal LP Basis

from an Interior Point Solution

Robert E. Bixby

Matthew J. Saltzman

October, 1991

TR91-32 



\title{
Recovering an Optimal LP Basis from an Interior Point Solution
}

\author{
Robert E. Bixby \\ Department of Mathematical Sciences \\ Rice University \\ Houston TX \\ 713/527-4805 bixby@rice.edu \\ Matthew J. Saltzman \\ Department of Mathematical Sciences \\ Clemson University \\ Clemson, SC \\ 803/656-3434rmjs@clemson.edu
}

Keywords: Programming (linear programming, interior point algorithms, simplex method, basis recovery); Analysis of algorithms (data structures, algorithms for basis recovery). 



\begin{abstract}
An important issue in the implementation of interior point algorithms for linear programming is the recovery of an optimal basic solution from an optimal interior point solution. In this paper we describe a method for recovering such a solution. Our implementation links a high-performance interior point code (OB1) with a high-performance simplex code (CPLEX). Results of our computational tests indicate that basis recovery can be done quickly and efficiently.
\end{abstract}



In recent years, interior point methods for linear programming have generated much excitement. High-performance interior point codes such as OB1 (OB1 is a trademark of XMP, Inc.) compete with advanced simplex codes such as CPLEX (CPLEX is a trademark of CPLEX, Inc.) to solve problems on a scale considered completely impractical only a few years ago. Currently, each class of methods outperforms the other on some types of problems, and neither has proved to be superior overall.

One important challenge in the implementation of interior point algorithms is the development of methods to construct an optimal basis. Whereas the simplex method naturally terminates with an optimal basic pair of primal and dual solutions, interior point algorithms provide only a "nearly" (strictly) complementary primal-dual pair. In fact, if multiple primal or dual optima exist, the interior point solution is likely be a point in the "middle" of the optimal face $[5,11]$. This is a significant drawback in some applications, since the current state of the art allows post-optimality analysis to proceed only from a basic primal-dual optimal pair. (See [1] for an investigation into the potential for post-optimality analysis in the context of interior point methods.)

Of particular interest is the reoptimization of a modified problem, given the optimal solution to the unmodified problem. This situation arises frequently in integer programming, where branching or adding cutting planes causes the last known solution to become infeasible. It is also an issue in nonlinear programming methods that solve sequences of LPs, and in many planning applications where problem structure remains the same, but coefficient values change from one period to the next.

An additional difficulty with current implementations of interior point methods is their numerical stability near the optimal solution. The linear system solved at each iteration of the Newton barrier algorithm implemented in $\mathrm{OB} 1$ is singular at a strictly complementary point, and as the solution is approached, the system becomes progressively more ill-conditioned. This may lead to problems such as difficulty meeting convergence criteria, or 
unacceptably large residuals. Although future developments in interior point techniques may eventually overcome these difficulties, recovery of a basic optimum is currently the best method available to ensure accuracy when the interior point methods encounter difficulties.

One early attempt to recover basic optima from interior optima by "crossing over" to the simplex method is described in [10]. This experiment applied an extension to Marsten's XMP simplex code to handle "superbasic" variables and combat stalling to the solution generated by a dual affine interior point algorithm. The dual affine algorithm produces a dual interior solution and a nearby complementary primal solution, which may not be primal feasible. The (then-current) extended XMP accepted a superbasic solution (see below), and set up an all-logical basis. Variables near their bounds were locked at their bounds, and the simplex method was started. After an optimal solution was found, the locked variables were released, and the problem was reoptimized. Finally, any remaining superbasic variables were moved to their bounds or pivoted into the basis. This experiment met with decidedly mixed success. Overall, the basis recovery phase took about $45 \%$ of the interior point time and about $30 \%$ of the time required to solve the problems from scratch using XMP's simplex algorithm. On some problems, basis recovery took longer than the interior-point phase.

The approach described in this paper is philosophically similar to that of [10], but it starts with the output of a primal-dual interior algorithm, generates an advanced starting basis, and uses different rules for choosing and manipulating superbasic variables. We demonstrate that the combination of simple crossover rules, switch from a primal-dual interior algorithm and a robust simplex implementation yields a very effective basis recovery method. The critical factors in designing a successful crossover are the identification of the positive variable in a primal-dual complementary pair, and the construction of a stable starting basis.

We begin in Section 1 with a review of essential concepts. In Section 2, we describe the rules for selecting variables to appear in a proposed starting 
basis. In Section 3, we describe the techniques we apply to repair the initial basis in case the proposed basis is invalid. Section 4 describes the pivoting strategy to move to a basic optimum. Recent results by Megiddo [12] have shown that, given a complementary primal-dual optimal pair, there is a strongly polynomial algorithm for basis recovery. In Section 5 , we describe an interpretation of Megiddo's result and compare this method with our proposal. Finally, Section 6 describes a comprehensive set of tests on the Netlib test set [3], and Section 7 presents final remarks.

\section{Basic Concepts}

We consider the bounded-variable linear program (LP):

$$
\begin{aligned}
\text { Minimize } & c^{T} x \\
\text { subject to } & A x=b \\
& l \leq x \leq u
\end{aligned}
$$

where $A$ is an $m \times n$ matrix. Arbitrary bounds, including $l_{j}=-\infty$ and $u_{j}=\infty$, are allowed.

When presented with a problem in this form, $\mathrm{OB} 1$ transforms it into a problem in which all variables satisfy $0 \leq x_{j} \leq u_{j}$, with $u_{j}=\infty$ allowed. The problem is transformed back to its original form before it is handed off to the crossover routine.

A normal basic solution to (1) is described by a partition of the index set $\{1, \ldots, n\}$ into subsets $\mathcal{B}, \mathcal{L}, \mathcal{U}$ and $\mathcal{F}$, such that the following properties hold (for convenience, we define $\mathcal{N}=\mathcal{L} \cup \mathcal{U} \cup \mathcal{F}$ ):

- $|\mathcal{B}|=m$ and the submatrix $B$ of $A$, consisting of the columns $\left\{A_{\cdot j} \mid j \in\right.$ $\mathcal{B}\}$ is nonsingular.

- $\mathcal{L} \subseteq\left\{j \in \mathcal{N}: l_{j}>-\infty\right\}$

- $\mathcal{U} \subseteq\left\{j \in \mathcal{N}: u_{j}<\infty\right\}$ 
- $\mathcal{F}=\left\{j \in \mathcal{N}: l_{j}=-\infty\right.$ and $\left.u_{j}=\infty\right\}$.

The variables are assigned the values

$$
\begin{aligned}
& x_{j}=\left\{\begin{array}{ll}
l_{j} & j \in \mathcal{L} \\
u_{j} & j \in \mathcal{U} \\
0 & j \in \mathcal{F}
\end{array} \text { for all } j \in \mathcal{N}\right. \\
& x_{B}=B^{-1}\left(b-L x_{L}-U x_{U}-F x_{F}\right)=B^{-1}\left(b-N x_{N}\right)
\end{aligned}
$$

(where $L, U, F$ and $N$ are defined similarly to $B$ ).

A superbasic solution to (1) is described by a similar partition, with the following modification:

- $j \in \mathcal{F}$ is permitted for any $j$ such that $l_{j}<u_{j}$, and for $j \in \mathcal{F}$, any value for $x_{j}$ is permitted, as long as $l_{j}<x_{j}<u_{j}$.

- the solution is specified by the sets $\mathcal{B}, \mathcal{L}, \mathcal{U}$ and $\mathcal{F}$, and the value of $x_{F}$. The value of $x_{B}$ is computed as above.

Note that any feasible solution to (1) can be described as a superbasic solution involving any basis $\mathcal{B}$ (with $B$ nonsingular), and suitably-chosen $\mathcal{L}, \mathcal{U}$, $\mathcal{F}$ and $x_{F}$.

\subsection{The Interior Point Algorithm}

The interior point code used in this experiment is OB1, which implements a primal-dual barrier method with a predictor-corrector enhancement. The details of the algorithm are described in [9], and the details of the Cholesky factorization are described in [7]. Reordering of the rows of $A$ to control fillin in the factorization of $A A^{T}$ is accomplished using the Multiple Minimum Degree heuristic [8]. The only modification to OB1 itself was the substitution of our own crossover routine for the routine which crosses over from the interior-point phase to the XMP simplex code. 


\subsection{The Simplex Algorithm}

The simplex code we use is CPLEX, modified to handle superbasic primal variables. These are treated as free variables, except that, if one is selected to enter the basis, the appropriate bound participates in the distance computation for selecting a leaving variable, and it may move to that bound without requiring a pivot.

At the end of a simplex run, any remaining superbasic variables are forced to move to a bound or enter the basis, and free variables are forced to enter the basis or move to zero. CPLEX terminates with a normal basic feasible solution.

Code to accept a starting superbasic solution and generate a usable starting basis is included, and this is described in detail below. The superbasic code uses partial/multiple reduced gradient pricing or a hybrid strategy mixing Devex [6] and partial/multiple pricing, and handles structural or slack variables between their bounds. Variables that violate their bounds are permitted only if they are in the basis.

\section{Selecting a Candidate Basis}

The first step in our crossover process is to identify which primal variables are to be considered away from their bounds. This is accomplished as follows:

- For each $x_{j}$ such that $l_{j}<u_{j}$, we compute $s_{j}=\min \left\{x_{j}-l_{j}, u_{j}-x_{j}\right\}$. The variables are sorted in decreasing order of $s_{j}$. If $x_{j}$ is free, then $s_{j}=\left|x_{j}\right|$. Fixed variables are excluded from consideration.

- Variables are examined in order of decreasing $s_{j}$. A variable $x_{j}$ is considered to be between its bounds as long as $s_{j}>\epsilon_{1}$, where $\epsilon_{1}=$ $10^{-5}$ was used in all our computational tests.

- The first $m$ such variables are marked as candidates for the initial basis. Any remaining variables with $s_{j}>\epsilon_{2}$ are marked as superbasic, 
where $\epsilon_{2}=10^{-4}$ in our tests. The case where fewer than $m$ candidates are available is treated below. All variables with $s_{j} \leq \epsilon_{2}$ are marked as being at the corresponding upper or lower bound, whichever is closer to the current value.

The status lists thus constructed are used as a starting point in the construction of the initial basis. Once an acceptable starting basis has been generated, the remaining superbasic variables are checked again, and set at the nearest bound if $x_{j} \leq \epsilon_{2}$.

\section{Forming the Starting Basis}

As we have pointed out, any superbasic solution can be described in terms of any basis. Our task is to choose a basis in which to describe the interior solution. This basis should contain a maximal set of variables strictly away from their bounds. The difficulty is that when we compute $x_{B}$ as the solution to $B x_{B}=b^{\prime}=b-N x_{N}$, the resulting residual $b^{\prime}-B x_{B}$ may be acceptably small (since Gaussian elimination effectively minimizes the residual), but the computed $x_{B}$ may not accurately approximate the $x_{B}$ that is passed from the interior algorithm. Even if the residual is within acceptable limits, $x_{B}$ may be highly sensitive to pertubations caused by moving nonbasic variables to bounds. (See [4], for example, for a discussion of these issues.) An inaccurately-computed $x_{B}$ may appear to have large infeasibilities (with respect to bounds), and may cause other numerical difficulties for the simplex algorithm. Thus it is extremely important to construct a very well-conditioned basis, even if this means rejecting variables that appear desirable, and that would be acceptable in other circumstances. We construct such a basis using the method described in this section.

The interior solution and the status list constructed above are used to generate a candidate basis (partial basis, in case fewer than $m$ candidates are found). CPLEX attempts to factor this matrix, as described in [2] (see also [14]). 
One aspect of the factorization that is of interest here is the singularity tolerance, $\tau$. The LP is scaled by CPLEX as part of its initialization, so that $\max _{i, j}\left|a_{i j}\right| \leq 1$. The singularity tolerance gives the minimum absolute magnitude of an acceptable pivot entry. For this initial factorization attempt, we select $\tau=10^{-3}$ (compared to the default of $\tau=10^{-8}$ for normal operation). Columns for which no acceptable pivot can be found are rejected. Each of the remaining columns is said to cover the row in which its pivot entry appears.

If the basis is incomplete, either because of an insufficient number of candidates or because of rejected columns, it is extended as follows: any non-basic slack variable that covers an uncovered row is included; other candidates are taken from the list of variables initially marked superbasic (not including rejected variables), if any, then from the variables at bounds, according to criteria described in [2]. The factorization is attempted again. Any uncovered rows after this pass are covered by artificial variables. The factorization and addition of artificial columns may be repeated up to ten times, if required to produce a complete, successfully factored basis.

Once a basis has been successfully constructed, the value of $x_{B}$ is computed, and the resulting solution becomes the initial candidate starting superbasic solution. If the total scaled infeasibility of this solution is greater than 1.0, this is taken as an indication that the chosen basis is too illconditioned to be acceptable. In this case, the entire process is repeated, starting with the current candidate basis, with $\tau=10^{-1}$.

The test for infeasibility after the first successful factorization (with $\tau=10^{-3}$ ) essentially approximates a test for the accuracy of the computed $x_{B}$. Although this test does not distinguish between infeasibilities due to inaccuracy and infeasibilities due to superbasic variables being pushed to bounds, it is quite effective in practice. If the infeasibility is small enough, we accept the current basis. If not, we demand a better-conditioned basis, by applying the selection process again with $\tau=10^{-1}$. If the new solution is still not satisfactory, then the infeasibility is probably not due 
to ill-conditioning, and we proceed using the solution in any case. An im-

provement to this method would be to use large infeasibility as an indicator to adjust both $\tau$ and $\epsilon_{2}$, i.e., to attempt to control infeasibility due to illconditioning as well as movement of nonbasic variables. These issues remain a topic for future investigation.

\section{Progress Toward a Normal Basic Optimum}

After an acceptable basis has been constructed and variables moved to bounds as described above, the remaining nonbasic variables are made superbasic at their interior-solution values. Then $x_{B}$ and the reduced costs corresponding to the current basis are computed. Because of the adjustments to the values of $x_{L}$ and $x_{U}$, the computed values of $x_{B}$ may be slightly different from their interior-solution values, and may even no longer be feasible. Also, the reduced costs associated with the chosen basis may not satisfy the optimality condition. Nevertheless, the superbasic solution we have constructed can be used to start phase I of the simplex method.

We apply CPLEX's implementation of Wolfe's piecewise-linear phaseI algorithm [15] (if necessary), beginning with the constructed superbasic solution. When a primal feasible solution is reached, we apply CPLEX's phase-II algorithm using partial and multiple pricing, or a hybrid of partial and multiple pricing and Devex pricing [6] (see Section 6 for a comparison). A superbasic candidate to enter the basis is treated the same way as a free variable, except that it may reach a bound before any basic variable, in which case it is moved into $\mathcal{L}$ or $\mathcal{U}$, as appropriate, rather than entering the basis.

When all feasibility and optimality conditions are satisfied, there may remain superbasic variables with reduced cost close enough to zero to be considered optimal. The last phase of the algorithm moves each such variable in turn to a bound: if the variable has only one bound, it is moved there; if it has upper and lower bounds, it is moved to the nearest bound (and to 
the other bound if the pivot is rejected). Free variables are moved to zero. During each step of this process, if a basic variable reaches its bound, it is exchanged in the basis with the superbasic variable being moved. This step is performed last, because superbasic variables often move during the reoptimization phase anyway. Every such movement results in the removal of one superbasic variable from the list. Thus, there may be significantly fewer superbasic variables at the end of the reoptimization process than at the beginning.

\section{Complexity and Megiddo's Method}

In [12], Megiddo describes a strongly polynomial algorithm for recovering an optimal basic solution from any complementary primal and dual feasible pair of solutions. He also shows that, if a strongly polynomial algorithm existed for recovering a basic optimal solution from a primal or dual optimal solution alone, then there would be a strongly polynomial algorithm for the general LP problem.

Given an exact complementary pair of primal and dual optimal solutions, Megiddo proposes inserting variables into the basis one at a time, as follows (for simplicity, we assume in this section that $0 \leq x_{j} \leq \infty$ ):

1. Insert positive primal variables until a maximal linearly independent set of columns has been included. Each time a column corresponding to a positive variable is found to be linearly dependent on columns already in the basis, move the variable to zero or pivot it into the partial basis if a basic variable goes to zero first. After this step all positive variables have been inserted in the basis or set to zero.

2. If any zero-valued variables have duals equal to zero, include as many as possible with columns linearly independent of those in the partial basis. 
3. Attempt to include each of the remaining variables until a complete basis has been constructed. For each zero-valued variable included with positive dual, move the dual to zero. If a nonbasic dual (with respect to the primal basis) goes to zero, pivot the corresponding variable into the partial basis.

When these steps are completed, a complete basis has been constructed, all basic dual variables are zero and all nonbasic primal variables are zero. Since (under exact arithmetic), every step increases the size of the basis or reduces the number of positive nonbasic variables, it follows that the number of iterations is bounded by $n$.

Megiddo's method differs from the method presented here in two ways. First, rather than build the basis one column at a time, we construct a full basis at the start and use the usual simplex pivoting mechanism to recover our solution. Megiddo's algorithm can be easily modified to accommodate this strategy, as described below. Second, our method does not make use of the dual solution provided. We discuss the implications of each of these differences next.

Meggido's method can be modified to start by constructing a full basis, then use simplex pivots. Starting with a complementary primal-dual pair of solutions, perform the following steps:

1. Find a maximal linearly independent set of columns corresponding to variables with positive primal values (i.e., dual values of zero). Any remaining positive primal variables will be superbasic.

2. Fill out the basis with any linearly independent columns from among the remaining variables. First include variables with primal and dual values both zero, then those with nonzero dual values.

3. For each of the remaining superbasic variables, move the variable to a bound, or until a variable in the basis reaches a bound. If the latter occurs, exchange the variables. 
4. For each basic variable with positive dual value, move the dual value to zero, or until a nonbasic dual variable reaches zero (as in the dual simplex method). If the latter occurs, exchange the variables.

At the completion of step 2, a basis matrix has been constructed, with respect to which the original solutions are primal- and dual-superbasic, i.e., some nonbasic primal variables may be positive, and some basic dual variables may be positive. After each iteration in step 3 , there will be one fewer nonbasic positive primal variable. If the exchange occurs, the incoming variable must replace a variable in the basis with a positive value, since the basis contains a maximal linearly independent set of these. At the conclusion of step 3, the primal solution is basic and feasible, but the corresponding reduced costs may violate the optimality conditions. At this point the original dual solution may be viewed as a superbasic starting solution for the dual simplex method. Each iteration of step 4 reduces the number of nonzero basic dual variables by one, without sacrificing dual feasibility. At the conclusion of this step, the algorithm will produce a normal basic optimal solution to the LP. (Note: if there are nonbasic free variables, they can be moved to zero or pivoted into the basis in step 3.) The algorithm is guaranteed to run in at most $n$ iterations in steps 3 and 4 .

The other difference of our method from Megiddo's method is that we do not make use of the dual solution provided. Megiddo shows that a basisrecovery algorithm using only primal or dual information could not have strongly polynomial worst-case running time unless a strongly polynomial algorithm for the general LP problem exists. Nevertheless, we will show that our algorithm performs comparatively well in practice. Although we have not implemented the superbasic dual simplex method, we can still use Megiddo's algorithm as a suggestive standard for comparison. This is because once a starting basis has been constructed, the number of pivots required by the algorithm (under the assumption of ideal arithmetic) can be easily estimated. What cannot be estimated is the fraction of primal and dual pivots that will require an exchange of variables in the basis. This can 
have a significant impact on the total running time of the algorithm, since pivots requiring an exchange are more costly. On the other hand, there is no a priori reason to expect this fraction to be lower than for our method.

It is unlikely that Megiddo's method could be implemented in a way that meets the guarantees of the analysis, for two reasons. The theoretical analysis begins with an exact optimal primal-dual pair, and assumes that the set of primal variables not at bounds can be identified precisely. Any practical implementation will start with an interior solution that is only approximately complementary (and perhaps only approximately feasible). Adjusting values of primal and dual variables to achieve complementarity may adversely affect primal or dual feasibility. The theoretical analysis also assumes that linear independence is a strictly combinatorial notion. Again, this cannot be true for linear systems represented as floating point numbers on digital computers. The condition of the basis is a critical problem, and nonsingular matrices can appear to be numerically singular or vice versa. If incorrect decisions are made with regard to inclusion of variables in the basis, a maximal set of linearly independent positive variables may not be included. In this eventuality, reduction in the number of primal or dual superbasic variables can no longer be guaranteed. If the basis is ill-conditioned, the computed solution may not be accurate (or feasible) and ratio tests may not be performed accurately.

For each of the problems we solved, we estimated the number of Megiddo pivots to be expected under ideal conditions. The estimate was computed, after a satisfactory initial basis had been constructed, as the number of superbasic primal variables plus the number of superbasic dual variables. This number is reported together with the times and number of pivots required by our algorithm, in Tables IV-VI. We observe that on only 14 problems does the number of actual pivots performed by our method exceed the number predicted. Overall, the number of pivots predicted exceeds the number actually performed by about $37 \%$. If we assume that the average cost of an iteration is the same for both methods on each problem, the projected total 
solution time from Megiddo's method is higher by $47 \%$ than the actual time. As the predictions are for ideal conditions, we can expect that the actual cost for an implementation of Megiddo's method using the same starting basis would be even higher.

It is apparent that, in most cases, our algorithm requires significantly fewer pivots than the Megiddo method. We might take this to be another instance of a familiar syndrome in linear programming: that algorithms with worst-case behavior that is polynomial often exhibit their worst-case behavior, whereas more daring algorithms may perform better on most practical problems, even if their worst-case behavior is not guaranteed to be good. On the other hand, there are likely to be certain problems for which Megiddo's method will outperform our method by a significant margin (in our test set, $\operatorname{wood} 1 p$ is a potential example). It is not apparent how to make the distinction a priori.

\section{Computational Tests}

We tested the proposed basis recovery algorithm on the problems in the Netlib test set [3]. Tests were run on a Sun $4 / 490$ with 64 megabytes of RAM, running SunOS 4.1.1. OB1 was compiled using the Sun $f 77$ compiler, version 1.3.1, and the command line performance options -04-cg89 -dalign -libmil. CPLEX was compiled with the Sun cc compiler supplied with the operating system, using the same command line options.

Two complete sets of tests were run, using different termination criteria for the interior point phase. In both tests, the default settings for OB1's parameters were used, except:

- The problem fit2p could not be solved using a reasonable amount of memory without resorting to a technique for handling dense columns. We used the OB1 parameter dense 10, which effectively removes all 25 columns in this model with more than one nonzero element from the Cholesky factorization, and handles them separately using a Schur 
complement technique [9].

- In the first set of tests, OB1 was terminated when the relative duality gap reached the default tolerance of $10^{-8}$. To test the effect of a more accurate interior solution, the second set of tests was run until the duality gap reached $10^{-12}$. OB1 occasionally terminated the high-accuracy run due to numerical difficulties encountered before the desired accuracy was achieved. In problems perold, fffff800 and nesm, the obejctive function values differed from the standard-accuracy run by $10^{-8}$.

In all cases, the CPLEX parameters were left at their defaults, except for the choice of pricing strategy. The results of comparisons among these choices are discussed below.

Tables I-III list the vital problem statistics and the results of the OB1 runs. The original number of rows, columns and nonzeros in the constraint matrix is given, as well as the number of rows, columns and constraint nonzeros after preprocessing by OB1's crush procedure. The total CPU time for preprocessing includes the crush phase plus the time for reordering rows of the constraint matrix to control fill-in in the Cholesky step. The number of iterations, CPU seconds, objective function value, and a measure of how strict the complementarity of the solution is, is given for the standard accuracy run. Individual problem statistics are not given for the high-accuracy run. The results are summarized below. The complementarity measure is computed by calculating the largest $p$ such that the floating point value $a+10^{-p} b=a$, where $a$ and $b$ are the larger and smaller of $s_{j}$ and $z_{j}$ (the reduced cost), respectively. The ratio of the minimum $10^{-p}$ to machine epsilon is given in the table. Values close to one indicate difficulty determining whether $s_{j}$ or $z_{j}$ should be considered nonzero.

Tables IV-VI list the results of the CPLEX phase. Times are divided between "setup time," which includes loading and scaling the problem and constructing the starting basis, and "solve time," which includes phase I 
and II pivots, plus the pivots to eliminate remaining superbasic variables. The pivot counts are given, as well as the number of pivots which involved an exchange of variables in the basis and the number which involved moving a nonbasic variable to a bound, for the simplex and superbasic cleanup phases. The estimated number of Megiddo primal and dual pivots is also listed. For the setup phase, the number of factorizations is listed. Where two numbers are listed in this column, the first is the number of factorizations with $\tau=10^{-3}$, and the second is the number with $\tau=10^{-1}$.

The total time for OB1 for the entire test set at default accuracy (including setup and solution time) is 6063 seconds, and the total CPLEX time (including setup and solution time) is 706 seconds, or $11.6 \%$ of the total interior point time. For the high-accuracy interior point run, the total OB1 time is 7426 seconds and the total CPLEX time is 587 seconds or $7.9 \%$ of the interior point time. The overall times are 6769 seconds for standard accuracy and 8013 seconds for high accuracy. Thus, the more accurate interior point solution is helpful for the basis recovery phase, but the additional cost of obtaining the high-accuracy solution to begin with more than offsets the savings in the basis-recovery phase. Note that over half the CPLEX times are due to one problem, stochfor3. If this problem is dropped from the test set, basis recovery time drops to only $5 \%$ of interior point time. Our experience with stochfor 3 is described below.

These results can be contrasted with the results of [10] on a subset of 26 of these problems. The overall cost of basis recovery in that paper was $46 \%$ of the total interior point time. For the same subset of problems, the method presented here takes $18 \%$ of the interior point time. Furthermore, the dual affine interior algorithm and quotient minimum degree reordering heuristic of [10] are significantly slower than the implementation in OB1. In fact, it is interesting to point out that the total dual affine time for the tests in [10] was 8075 seconds, and the total recovery time was 3731 seconds (on a VAX 8600 with no floating point accelerator). The same test on the Sun $4 / 490$ using OB1's primal-dual predictor-corrector ran in 385 seconds and 
the CPLEX basis recovery time was 69 seconds. Although the effect of the change of machine is not clear, the total test now runs over 25 times faster than the earlier version. The interior point portion of the test is 21 times faster, and the recovery portion is 54 times faster.

\subsection{Perturbation}

Of the total 700 seconds reported in the previous section for basis recovery on the Netlib set, 370 seconds are attributable to two problems. Degen? took 918 iterations and 35 seconds and stocfor 3 took 2303 iterations and 335 seconds. With these two problems removed, the total basis-recovery time would have been 330 seconds, and the total OB1 time would have been essentially unchanged.

The behavior of stocfor 3 can explained as follows (degen 3 is similar). This problem has a basis of size 16675 . Using any sort of reasonable tolerance for zero (anything between $10^{-5}$ and $10^{-10}$ ), there are 14932 positive primal variables (including slacks) and all other variables (including slacks) have corresponding dual variables that are positive. Thus, the initial basis must necessarily contain $1743=16675-14932$ variables with positive dual values. Using Megiddo's procedure, these would take a minimum of 1743 iterations to correct. That would be an improvement over the 2303 iterations reported above, although the Megiddo iterations would likely be more expensive, and the total recovery time would still be close to the reported 330 seconds.

One approach for dealing with this problem is to introduce a perturbation, as follows. For each structural variable $x_{j}$, we replace the bounds on $x_{j}$ by $l_{j}-L_{j} \epsilon$ and $u_{j}+U_{j} \epsilon$, respectively, where $L_{j}$ and $U_{j}$ are independent uniform $[0,1]$-random variables, and $\epsilon$ is some specified tolerance (say $10^{-4}$ ). The resulting perturbed problem, $\overline{L P}$, is then given to $\mathrm{OB} 1$, and solved, and the solution passed to CPLEX. Basis recovery is carried out for $\overline{L P}$ using this solution. Where $\mathcal{B}$ is the recovered basis, $\mathcal{B}$ is used as the starting point for a simplex solution of the original problem. The expectation is that this $\mathcal{B}$ will be already be optimal for the original problem, and that no additional 
iterations will be required. The idea of the perturbation (which is essentially that already used in CPLEX for dealing with stalling in the normal simplex method) is to make not only the optimal solution a vertex, but to put the vertices in one-to-one correspondence with the basic feasible solutions. This approach differs from that proposed by Mehrotra [13], who perturbs only the objective, thus producing a unique vertex solution, but not a unique basic optimal solution.

For stocfor 3 this perturbation approach reduces the basis recovery time to 59 seconds (and 283 iterations). For degen 3 it reduces the time to 7.5 seconds (and 50 iterations). However, for several other problems that were tested, perturbation led to significant numerical difficulties for OB1, which could not then solve these problems to acceptable accuracy. This latter behavior has yet to be explained.

\section{Concluding Remarks}

We have described an effective method for recovering an optimal basis from an optimal primal/dual interior point solution. In tests on the Netlib problem set, this method was able to recover an optimal basis in $5 \%$ of the interior point solution for most problems. Although highly primal degenerate problems such as stocfor 3 appear to be intrinsically difficult, we have also indicated that pertubating the problem may effectively eliminate this difficulty. More experimentation is warranted, both in this area and in the selection of a robust starting basis. Nevertheless, it is clear that, in general, basis recovery can be done in reasonable time. For problems where the interior method is indicated, the requirement for an optimal basic solution need not be considered a significant drawback.

\section{References}

[1] I. Adler and R. D. C. Montiero (1989), A Geometric View of Para- 
metric Linear Programming, Unnumbered Technical Report, Dept. of Industrial Engineering and Operations Research, University of California, Berkely CA.

[2] R. E. Bixby (1990), Implementing the Simplex Method: The Initial Basis, Technical Report TR90-32, Dept. of Mathematical Sciences, Rice University, Houston TX.

[3] D. M. Gay (1985), "Electronic Mail Distribution of Linear Programming Test Problems," COAL Newsletter 13, December, 10-13.

[4] P. E. Gill, W. Murray and M. H. Wright (1991), Numerical Linear Algebra and Optimization v/1, Addison Wesley, Redwood City CA.

[5] O. Güler and Y. Ye (1991), Convergence Behavior of Some InteriorPoint Algorithms, Working Paper Series No. 91-4, Dept. of Management Sciences, The University of Iowa, Iowa City, Iowa.

[6] P. M. J. Harris (1973), "Pivot Selection Methods in the Devex LP Code," Mathematical Programming 5, 1-28.

[7] H.-W. Jung, R. E. Marsten and M. J. Saltzman (1991), The Column Cholesky Method for Numerical Factorization in Interior Point Algorithms, Technical Report \#596, Dept. of Mathematical Sciences, Clemson University, Clemson SC.

[8] J. W.-H. Liu (1985), "Modification of the Minimum-Degree Algorithm by Multiple Elimination," ACM Transactions on Mathematical Software 11:2, June, 141-153.

[9] I. J. Lustig, R. E. Marsten and D. F. Shanno (1990), On Implementing Mehrotra's Predictor-Corrector Interior Point Method for Linear Programming, Technical Report SOR90-03, Dept. of Civil Engineering and Operations Research, Princeton University, Princeton NJ. 
[10] R. E. Marsten, M. J. Saltzman, D. F. Shanno, G. S. Pierce and J. F. Ballintijn (1989), "Implementation of a Dual Affine Interior Point Algorithm for Linear Programming," ORSA Journal on Computing 1:4, $287-297$.

[11] N. Megiddo (1989), "Pathways to the Optimal Set in Linear Programming," in N. Megiddo, ed., Progress in Mathematical ProgrammingInterior Point and Related Methods, Springer-Verlag, New York, 131158.

[12] N. Megiddo (1991), "On Finding Primal- and Dual-Optimal Bases," ORSA Journal on Computing 3:1, 63-65.

[13] S. Mehrotra (1990), On Finding a Vertex Solution Using Interior Point Methods, Technical Report 89-22, Dept. of Industrial Engineering and Management Sciences, Northwestern University, Evanston IL.

[14] U. H. Suhl and L. M. Suhl (1990), "Computing Sparse LU Factorizations for Large-Scale Linear Programming Bases," ORSA Journal on Computing 2:4, 325-335.

[15] P. Wolfe (1965), "The Composite Simplex Algorithm," SIAM Review 7:1, 42-54. 


\begin{tabular}{|c|c|c|c|c|c|c|c|c|c|c|c|}
\hline \multirow[b]{2}{*}{ name } & \multicolumn{3}{|c|}{ before crush } & \multicolumn{3}{|c|}{ after crush } & rep & \multicolumn{2}{|c|}{ solve } & \multirow[b]{2}{*}{ objval } & \multirow[b]{2}{*}{ compl } \\
\hline & $m$ & $n$ & $n z$ & $m$ & $n$ & $n z$ & secs & secs & iter & & \\
\hline afiro & 27 & $\overline{32}$ & 83 & 25 & $\overline{32}$ & 81 & 0.03 & $\overline{0.07}$ & 9 & $-4.64753140 \mathrm{e}+02$ & $1 \mathrm{e}+03$ \\
\hline & & 48 & 118 & 48 & 48 & 118 & 03 & & 8 & & \\
\hline & 50 & 48 & 130 & 49 & 48 & 130 & 02 & & 10 & & \\
\hline & & 103 & 280 & 104 & 103 & 280 & & & 10 & & \\
\hline & & 41 & 286 & 43 & 41 & 286 & & & 15 & & \\
\hline & 56 & 97 & 383 & 53 & 97 & 380 & 0.05 & 0.26 & 12 & & \\
\hline & 129 & 140 & 420 & 95 & 140 & 385 & 0.06 & & 12 & & \\
\hline & & 111 & 447 & 106 & 111 & & 00 & & 19 & & \\
\hline & & 83 & 491 & 72 & 83 & 489 & & & 14 & & \\
\hline & 205 & 203 & 551 & 203 & 202 & 550 & 0.10 & 0.63 & 11 & -5.2 & \\
\hline & 91 & 180 & 663 & 75 & 170 & 603 & $\overline{0.0 \varepsilon}$ & 0.36 & 10 & -2.6 & $1 e+04$ \\
\hline & 96 & 79 & & 93 & 79 & & & & 12 & & \\
\hline & 198 & 203 & 908 & 53 & 194 & 444 & U & & 13 & & \\
\hline & & 308 & 1078 & 134 & 308 & 1045 & 11 & & 16 & & \\
\hline & & 225 & & & 225 & & & & 20 & & \\
\hline & & & & & 43 & 1 & & & 14 & & \\
\hline & & 35 & 1426 & & 358 & 1312 & & & 14 & & \\
\hline & & 315 & 1429 & & 304 & 1165 & 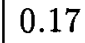 & 1.14 & 18 & & \\
\hline & & 500 & & & 500 & & & 2.01 & 16 & -1 & \\
\hline & 300 & 480 & 2 & 28 & 480 & & 17 & 1.75 & 15 & & $e+03$ \\
\hline & & & & & 367 & & 23 & 3.07 & 18 & & $e+02$ \\
\hline & & 249 & 148 & & 247 & & & & 19 & & \\
\hline & & 142 & & & 142 & & & 9.46 & 23 & -8 & \\
\hline & & 614 & 10 & & 607 & 2082 & & 4.37 & 24 & & \\
\hline & 616 & 1092 & & 590 & 10 & 23 & & & & & \\
\hline & 77 & & 388 & 77 & 760 & 2388 & & 15 & 11 & & \\
\hline & 400 & & 109 & & 669 & 2189 & & & 29 & -7 & \\
\hline & 48 & 163 & & & 163 & & & & 23 & & \\
\hline & 3 & 47 & & 25 & 467 & & & 51 & 17 & & $e+01$ \\
\hline & 223 & & 578 & 161 & 281 & & 0.8 & 2.70 & 22 & & $1 e+04$ \\
\hline & $\overline{330}$ & 457 & 2589 & & 457 & 2516 & 0.2 & & 17 & & \\
\hline & & & 2612 & & & & & & 14 & & -0 \\
\hline & 360 & 1183 & 3139 & 310 & 1064 & 2915 & & 08 & & & \\
\hline & 490 & 1169 & & 450 & 1165 & & & & 27 & & $1 e+02$ \\
\hline & 17 & 262 & & 113 & 26 & & & & & & \\
\hline & & 1775 & & & & & & & & & \\
\hline & 351 & 34 & 3485 & 308 & & & & 5.07 & 24 & -3 . & $1 e-01$ \\
\hline & 467 & 1075 & 36 & 409 & 1064 & & & & 24 & & +07 \\
\hline & & & & 35 & & & & 7.00 & 14 & & \\
\hline & & & & & & & & 8.05 & 14 & $-1.43517800 \mathrm{e}+03$ & $1 e+0$ \\
\hline
\end{tabular}

Table I: Test problem dimensions and interior point performance 


\begin{tabular}{|c|c|c|c|c|c|c|c|c|c|c|c|}
\hline \multirow[b]{2}{*}{ name } & \multicolumn{3}{|c|}{ before crush } & \multicolumn{3}{|c|}{ after crush } & prep & \multicolumn{2}{|c|}{ solve } & \multirow[b]{2}{*}{ objval } & \multirow[b]{2}{*}{ compl } \\
\hline & $m$ & $n$ & $n z$ & $m$ & $n$ & $n z$ & secs & secs & iter & & \\
\hline agg2 & 516 & 302 & 4284 & 458 & $\overline{302}$ & $\overline{4110}$ & 0.55 & 10.40 & 18 & $-2.02392520 \mathrm{e}+07$ & $1 e-01$ \\
\hline & 516 & 301 & 4300 & 458 & 302 & 4126 & 0.54 & 9.99 & 17 & $1.03121160 \mathrm{e}+07$ & $1 e+00$ \\
\hline & 147 & 1350 & 4316 & 147 & 1350 & 4316 & 0.22 & 2.43 & 12 & $5.05000000 \mathrm{e}+01$ & $1 e+02$ \\
\hline ip 04s & 402 & 1458 & 4352 & 241 & 1458 & 4157 & 0.41 & 3.09 & 15 & $1.79871470 \mathrm{e}+06$ & $1 e+03$ \\
\hline ba & 515 & 1028 & 4352 & 449 & 994 & 4205 & 2.06 & 78.32 & 19 & $1.57116000 \mathrm{e}+04$ & $1 e+03$ \\
\hline & 333 & 587 & 4520 & 284 & 583 & 4406 & 0.54 & 5.46 & 19 & $2.92147770 \mathrm{e}-01$ & $1 e+00$ \\
\hline & 161 & 421 & 4563 & 133 & 421 & 4558 & 0.28 & 3.78 & 21 & $-6.64218960 \mathrm{e}+02$ & $1 e+00$ \\
\hline & 643 & 1175 & 5121 & 564 & 1172 & 4976 & 0.70 & 13.00 & 27 & $1.97762960 \mathrm{e}+03$ & $1 e+01$ \\
\hline & 410 & 1000 & 5141 & 397 & 1088 & 7190 & 1.07 & 21.26 & 35 & $-2.58113930 \mathrm{e}+03$ & $1 e-01$ \\
\hline $\mathrm{sm} 2$ & 660 & 914 & 5183 & 564 & 914 & 5037 & 0.42 & 6.57 & 19 & $3.66602620 \mathrm{e}+04$ & $1 e+00$ \\
\hline & 300 & 645 & 5620 & 300 & 645 & 5620 & 0.41 & 4.71 & 16 & $-1.06870940 \mathrm{e}+08$ & $1 \mathrm{e}+03$ \\
\hline pe & 625 & 1376 & 6018 & 600 & 1453 & 7101 & 0.92 & 32.51 & 36 & $e+03$ & $1 e-01$ \\
\hline ff 800 & 524 & 854 & 6227 & 484 & 854 & 6170 & 0.95 & 15.81 & 28 & $5.55679570 \mathrm{e}+05$ & $1 e-01$ \\
\hline ship04l & 402 & 2118 & 6332 & 317 & 2118 & 6101 & 0.69 & 4.50 & 15 & $1.79332450 \mathrm{e}+06$ & $1 e-01$ \\
\hline & 1090 & 1880 & 6714 & 1033 & 1880 & 6489 & 0.71 & 10.03 & 20 & $1.72480710 \mathrm{e}+03$ & $1 e+05$ \\
\hline & 1309 & 1681 & 6912 & 1137 & 1681 & 6740 & 0.84 & 16.60 & 16 & $35740 \mathrm{e}+05$ & $1 e+00$ \\
\hline p08s & 778 & 2387 & 7114 & 326 & 2387 & 6305 & 0.64 & 4.01 & 14 & $e+06$ & $1 e+04$ \\
\hline rra & 1227 & 2036 & 7302 & 1212 & 2036 & 7272 & 0.65 & 12.02 & 18 & +07 & $1 e+00$ \\
\hline & 990 & 1371 & 7777 & 846 & 1371 & 7558 & 0.70 & 10.66 & 20 & +04 & $1 e+02$ \\
\hline & 1151 & 2763 & 8178 & 417 & 2763 & 7357 & 0.73 & 6.04 & 18 & $0 e+06$ & $1 e+03$ \\
\hline grow22 & 440 & 946 & 8252 & 440 & 946 & 8252 & 0.59 & 7.05 & 16 & $2+08$ & $1 \mathrm{e}+03$ \\
\hline stocfor2 & 2157 & 2031 & 8343 & 2141 & 2031 & 8319 & 1.14 & 20.73 & 22 & -3.90 & $1 e+04$ \\
\hline $\operatorname{scsd} 8$ & 397 & 2750 & 8584 & 397 & 2750 & 8584 & 0.47 & 4.30 & 10 & $9.05000000 \mathrm{e}+02$ & $1 e+05$ \\
\hline p3 & 1480 & 2480 & 8874 & 1408 & 2480 & 8595 & 0.91 & 12.23 & 17 & $1.42400000 \mathrm{e}+03$ & $1 e+02$ \\
\hline & 722 & 2789 & 9126 & 705 & 2863 & 9601 & 0.96 & 32.00 & 44 & $50 \mathrm{e}+06$ & $1 e-01$ \\
\hline & 846 & 1443 & 9614 & 680 & 1412 & 8653 & 1.05 & 17.07 & 31 & $-5.80637440 \mathrm{e}+04$ & $1 e+00$ \\
\hline & 627 & 1677 & 9868 & 627 & 1677 & 9868 & 4.02 & 446.95 & 16 & $e+03$ & $1 e+03$ \\
\hline $25 \mathrm{fv} 47$ & 821 & 1571 & 10400 & 780 & 1571 & 10360 & 1.15 & 33.86 & 25 & +03 & $1 \mathrm{e}+00$ \\
\hline & 929 & 3523 & 10669 & 689 & 3521 & 10126 & 2.31 & 16.36 & 35 & $0 e+06$ & $1 e+03$ \\
\hline & 778 & 4283 & 12802 & 520 & 4283 & 11614 & 1.29 & 8.56 & 16 & $520 \mathrm{e}+06$ & $1 e+03$ \\
\hline pilotnov & 975 & 2172 & 13057 & 870 & 2115 & 12514 & 1.76 & 51.8 & 20 & -4.4 & $1 \mathrm{e}+00$ \\
\hline & 662 & 2923 & 13288 & 646 & 2914 & 13256 & 0.91 & 31.09 & 30 & +07 & $1 e-01$ \\
\hline fit1d & 24 & 1026 & 13404 & 24 & 1026 & 13404 & 0.53 & 6.25 & 18 & $e+03$ & $1 e+03$ \\
\hline bnl2 & 2324 & 3489 & 13999 & 2113 & 3483 & 13690 & 2.76 & 161.39 & 32 & $1.81123650 \mathrm{e}+03$ & $1 \mathrm{e}+00$ \\
\hline & 940 & 1988 & 14698 & 831 & 2017 & 15354 & 2.13 & 119.81 & 43 & $-6.11313650 \mathrm{e}+03$ & $1 e+00$ \\
\hline ship12l & 1164 & 5427 & 16170 & 687 & 5427 & 14913 & 1.56 & 12.73 & 18 & $1.47018790 \mathrm{e}+06$ & $1 \mathrm{e}+00$ \\
\hline & 190 & 2857 & 20720 & 1528 & 2799 & 17293 & 2.68 & 68.08 & 30 & $00 \mathrm{e}+00$ & $1 e-01$ \\
\hline 80bau3b & 2262 & 9799 & 21002 & 2020 & 9768 & 20646 & 4.27 & 89.32 & 35 & $9.87224190 \mathrm{e}+05$ & $1 e-01$ \\
\hline & 1503 & 1818 & 24646 & 1503 & 1818 & 24646 & 48.91 & 138.76 & 18 & $-9.87294000 e+02$ & $1 e+01$ \\
\hline greenbea & 2392 & 5405 & 30877 & 1951 & 5296 & 28075 & 6.83 & 81.17 & 41 & $-7.25552480 \mathrm{e}+07$ & $1 e+00$ \\
\hline
\end{tabular}

Table II: Test problem dimensions and interior point performance (cont.) 


\begin{tabular}{|c|c|c|c|c|c|c|c|c|c|c|c|}
\hline \multirow[b]{2}{*}{ name } & \multicolumn{3}{|c|}{ before crush } & \multicolumn{3}{|c|}{ after crush } & prep & \multicolumn{2}{|c|}{ solve } & \multirow[b]{2}{*}{ objval } & \multirow[b]{2}{*}{ compl } \\
\hline & $m$ & $n$ & $n z$ & $m$ & $n$ & $n z$ & $\operatorname{secs}$ & secs & iter & & \\
\hline greenbeb & 2392 & 5405 & 30877 & 1947 & 5293 & 27746 & 6.77 & 65.11 & 34 & $-4.30226030 \mathrm{e}+06$ & $1 e-01$ \\
\hline $\mathrm{d} 2 \mathrm{q} 06 \mathrm{c}$ & 2171 & 5167 & 32417 & 2098 & 5167 & 32344 & 4.06 & 415.54 & 31 & $1.22784210 \mathrm{e}+05$ & $1 e-01$ \\
\hline woodw & 1098 & 8405 & 37474 & 711 & 8285 & 32695 & 2.63 & 36.72 & 20 & $1.30447630 \mathrm{e}+00$ & $1 e+00$ \\
\hline pilot & 1441 & 3652 & 43167 & 1391 & 3631 & 42974 & 11.90 & 568.91 & 29 & $-5.57489730 \mathrm{e}+02$ & $1 e-01$ \\
\hline fit $2 p$ & 3000 & 13525 & 50284 & 3000 & 13525 & 50284 & 2.22 & 76.27 & 18 & $6.84642930 \mathrm{e}+04$ & $1 e+01$ \\
\hline stocfor 3 & 16675 & 15695 & 64875 & 16643 & 15695 & 64819 & 9.53 & 267.09 & 35 & $-3.99767840 \mathrm{e}+04$ & $1 e-01$ \\
\hline woodlp & 244 & 2594 & 70215 & 171 & 2594 & 69422 & 3.11 & 25.72 & 14 & $-1.44290240 e+00$ & $1 e+00$ \\
\hline pilot87 & 2030 & 4883 & 73152 & 1991 & 4859 & 72967 & 34.97 & 2596.14 & 41 & $3.01710350 \mathrm{e}+02$ & $1 e-01$ \\
\hline fit $2 d$ & 25 & 10500 & 129018 & 25 & 10500 & 129018 & 5.38 & 84.85 & 24 & $-6.84642930 \mathrm{e}+04$ & $1 e+02$ \\
\hline total & & & & & & & 190.06 & 5873.03 & & & \\
\hline
\end{tabular}

Table III: Test problem dimensions and interior point performance (cont.) 


\begin{tabular}{|c|c|c|c|c|c|c|c|c|c|c|c|c|}
\hline \multirow[b]{3}{*}{ name } & \multicolumn{2}{|c|}{ setup } & \multicolumn{4}{|c|}{ solve } & \multicolumn{4}{|c|}{ pivot type } & \multirow{2}{*}{\multicolumn{2}{|c|}{$\frac{\text { Megiddo }}{\text { pivots }}$}} \\
\hline & \multirow[b]{2}{*}{ secs } & \multirow[b]{2}{*}{ fact } & \multirow[b]{2}{*}{ secs } & \multicolumn{3}{|c|}{ pivots } & \multicolumn{2}{|c|}{ simplex } & \multicolumn{2}{|c|}{ cleanup } & & \\
\hline & & & & ph. 1 & ph. 2 & cleanup & exch. & push & exch. & push & primal & dual \\
\hline afiro & $\overline{0.02}$ & 2 & 0.00 & 0 & $\overline{\overline{0}}$ & 2 & 0 & 0 & 1 & $\overline{1}$ & 2 & 7 \\
\hline sc50b & 0.01 & 1 & 0.02 & 0 & 0 & 0 & 0 & 0 & 0 & 0 & 0 & 0 \\
\hline sc50a & 0.03 & 2 & 0.02 & 0 & 1 & 0 & 1 & 0 & 0 & 0 & 0 & 3 \\
\hline 105 & 0.07 & 2 & 0.02 & 0 & 1 & 0 & 1 & 0 & 0 & 0 & 0 & 11 \\
\hline kb2 & 0.02 & 1 & 0.00 & 0 & 0 & 0 & 0 & 1 & 0 & 0 & 0 & 0 \\
\hline adlittle & 0.04 & 2 & 0.03 & 0 & 9 & 16 & 9 & 0 & 9 & 7 & 25 & 8 \\
\hline scagr7 & 0.01 & 1 & 0.02 & 0 & 2 & 0 & 0 & 2 & 0 & 0 & 2 & 0 \\
\hline stocfor 1 & 05 & 2 & 0.03 & 0 & 8 & 0 & 8 & 0 & 0 & 0 & 0 & 7 \\
\hline blend & 0.02 & 2 & 0.03 & 0 & 1 & 2 & 1 & 0 & 2 & 0 & 2 & 6 \\
\hline sc205 & 0.12 & 2 & 0.05 & 0 & 2 & 0 & 2 & 0 & 0 & 0 & 0 & 11 \\
\hline recipe & 0.04 & 2 & 0.03 & 0 & 3 & 58 & 3 & 0 & 17 & 41 & 59 & $\overline{3}$ \\
\hline share2b & 0.05 & 2 & 0.03 & 0 & 6 & 6 & 6 & 0 & 5 & 1 & 6 & 9 \\
\hline vtpbase & 0.07 & 2 & 0.00 & 0 & 0 & 0 & 0 & 0 & 0 & 0 & 0 & 1 \\
\hline lotfi & 0.08 & 2 & 0.05 & 0 & 7 & 28 & 7 & 0 & 10 & 18 & 30 & 8 \\
\hline are1b & 0.03 & 1 & 0.02 & 0 & 0 & 0 & 0 & 0 & 0 & 0 & 0 & 0 \\
\hline boeing2 & 0.03 & 2 & 0.07 & 0 & 16 & 13 & 16 & 0 & 8 & 5 & 13 & 30 \\
\hline scorpion & 0.13 & 2 & 0.17 & 0 & 30 & 0 & 30 & 0 & 0 & 0 & 0 & 22 \\
\hline bore3d & 0.08 & 2 & 0.05 & 0 & 6 & 0 & 6 & 0 & 0 & 0 & 2 & 10 \\
\hline $\operatorname{agr} 25$ & 0.13 & 2 & 0.17 & 0 & 32 & 0 & 30 & 2 & 0 & 0 & 32 & 72 \\
\hline sctap1 & 0.10 & 2 & 0.30 & 0 & 36 & 101 & 36 & 0 & 43 & 58 & 104 & 53 \\
\hline capri & 0.12 & 2 & 0.13 & $\overline{0}$ & 12 & 35 & 12 & 0 & 11 & 24 & 41 & 7 \\
\hline brandy & 0.15 & 2 & 0.10 & 1 & 3 & 5 & 3 & 0 & 2 & 3 & 7 & 9 \\
\hline israel & 0.10 & 2 & 0.13 & 1 & 10 & 19 & 11 & 0 & 9 & 10 & 30 & 11 \\
\hline finnis & 0.15 & 2 & 0.33 & 0 & 42 & 65 & 39 & 3 & 39 & 26 & 69 & 91 \\
\hline giffpin & 0.27 & 2 & 1.10 & 0 & 180 & 0 & 180 & 0 & 0 & 0 & 77 & 233 \\
\hline sd1 & 0.10 & 2 & 0.25 & 0 & 71 & 12 & 71 & 0 & 3 & 9 & 12 & 46 \\
\hline etamacro & 0.15 & 2 & 0.27 & 0 & 36 & 21 & 35 & 1 & 17 & 4 & 24 & 46 \\
\hline & 0.10 & 2 & 0.08 & 0 & 26 & 5 & 2 & 24 & 0 & 5 & 31 & 5 \\
\hline bandm & 0.16 & 2 & 0.02 & 0 & 0 & 0 & 0 & 0 & 0 & 0 & 0 & 2 \\
\hline $\mathrm{e} 226$ & 0.13 & 2 & 0.07 & 0 & 5 & 4 & 5 & 0 & 2 & 2 & 4 & 8 \\
\hline scfxm1 & 0.12 & 2 & 0.13 & 0 & 8 & 12 & 8 & 0 & 4 & 8 & 12 & 25 \\
\hline grow7 & 0.28 & 2 & 0.85 & 6 & 48 & 97 & 16 & 38 & 66 & 31 & 151 & 6 \\
\hline standata & 0.18 & 2 & 0.07 & 0 & 2 & 12 & 2 & 0 & 0 & 12 & 12 & 212 \\
\hline scrs8 & 0.23 & 2 & 0.17 & 0 & 5 & 16 & 5 & 0 & 16 & 0 & 16 & 111 \\
\hline beaconfd & 0.08 & 2 & 0.05 & 0 & 2 & 4 & 2 & 0 & 2 & 2 & 6 & 1 \\
\hline shell & 0.24 & 2 & 0.48 & 0 & 63 & 1 & 63 & 0 & 0 & 1 & 8 & 146 \\
\hline boeing1 & 0.19 & 2 & 0.18 & 0 & 20 & 15 & 20 & 0 & 8 & 7 & 18 & 32 \\
\hline standmps & 0.16 & 2 & 0.17 & 0 & 13 & 14 & 13 & 0 & 6 & 8 & 14 & 209 \\
\hline stair & 0.80 & 2 & 0.27 & 0 & 0 & 1 & 0 & 0 & 1 & 0 & 1 & 1 \\
\hline degen 2 & 0.15 & 2 & 2.23 & 0 & 242 & 0 & 242 & 0 & 0 & 0 & 0 & 171 \\
\hline
\end{tabular}

Table IV: Basis recovery performance 


\begin{tabular}{|c|c|c|c|c|c|c|c|c|c|c|c|c|}
\hline \multirow[b]{3}{*}{ name } & \multicolumn{2}{|c|}{ setup } & \multicolumn{4}{|c|}{ solve } & \multicolumn{4}{|c|}{ pivot type } & \multirow{2}{*}{\multicolumn{2}{|c|}{$\frac{\text { Megiddo }}{\text { pivots }}$}} \\
\hline & \multirow[b]{2}{*}{ secs } & \multirow[b]{2}{*}{ fact } & \multirow[b]{2}{*}{ secs } & \multicolumn{3}{|c|}{ pivots } & \multicolumn{2}{|c|}{ simplex } & \multirow{2}{*}{\multicolumn{2}{|c|}{$\begin{array}{l}\text { cleanup } \\
\text { exch. push }\end{array}$}} & & \\
\hline & & & & ph. 1 & ph. 2 & cleanup & exch. & push & & & primal & dual \\
\hline agg2 & $\overline{0.16}$ & 2 & 0.47 & $\overline{4}$ & 96 & 3 & 17 & $\overline{83}$ & 12 & 21 & 131 & 14 \\
\hline agg3 & 0.16 & 2 & 0.42 & 8 & 69 & 32 & 16 & 61 & 14 & 18 & 107 & 12 \\
\hline sd6 & 0.20 & $2 / 1$ & 0.30 & 0 & 40 & 78 & 40 & 0 & 12 & 66 & 80 & 40 \\
\hline ship04s & 0.25 & 2 & 0.15 & 0 & 12 & 1 & 12 & 0 & 0 & 1 & 1 & 27 \\
\hline seba & 0.20 & 2 & 0.07 & 0 & 1 & 0 & 1 & 0 & 0 & 0 & 0 & 9 \\
\hline tuf & 0.15 & 2 & 0.30 & 0 & 25 & 51 & 25 & 0 & 16 & 35 & 51 & 113 \\
\hline forplan & 0.13 & 2 & 0.10 & 0 & 15 & 0 & 15 & 0 & 0 & 0 & 2 & 26 \\
\hline & 0.35 & 2 & 1.63 & 21 & 54 & 227 & 74 & 1 & 72 & 155 & 292 & 76 \\
\hline lot4 & 0.83 & 3 & 0.90 & 8 & 12 & 34 & 20 & 0 & 3 & 31 & 42 & 19 \\
\hline scfxm2 & 0.30 & 2 & 0.43 & 0 & 23 & 23 & 23 & 0 & 8 & 15 & 31 & 41 \\
\hline grow15 & 0.81 & 3 & 3.32 & 17 & 5 & $\overline{233}$ & 22 & 0 & 162 & 71 & 254 & 8 \\
\hline rold & 1.12 & 4 & 2.58 & 2 & 71 & 37 & 68 & 5 & 19 & 18 & 57 & 29 \\
\hline $\mathrm{f} 800$ & 0.25 & 2 & 0.48 & 2 & 36 & 51 & 28 & 10 & 19 & 32 & 89 & 166 \\
\hline ship04l & 0.33 & 2 & 0.37 & 0 & 29 & 1 & 29 & 0 & 1 & 0 & 1 & 45 \\
\hline sctap2 & 0.40 & 2 & 1.53 & 0 & 58 & 230 & 58 & 0 & 34 & 196 & 230 & 249 \\
\hline nges & 0.77 & 3 & 3.08 & 148 & 0 & 147 & 148 & 0 & 87 & 60 & 286 & 0 \\
\hline p08s & 0.37 & 2 & 0.38 & 0 & 27 & 0 & 27 & 0 & 0 & 0 & 0 & 68 \\
\hline rra & 0.48 & 2 & 1.10 & 0 & 68 & 12 & 66 & 2 & 1 & 11 & 26 & 234 \\
\hline scfxm3 & 0.41 & 2 & 0.67 & 0 & 22 & 34 & 22 & 0 & 12 & 22 & 34 & 46 \\
\hline ship12s & 0.45 & 2 & 0.48 & 0 & 38 & 0 & 38 & 0 & 0 & & 0 & 76 \\
\hline grow22 & 1.28 & 3 & 7.60 & 20 & 0 & 409 & 20 & 0 & 271 & 138 & 429 & 0 \\
\hline stocfor 2 & 0.70 & 2 & 3.73 & 0 & 196 & 0 & 196 & 0 & 0 & 0 & 0 & 195 \\
\hline $\operatorname{scsd}$ & 0.49 & $2 / 1$ & 1.53 & 0 & 55 & 174 & 55 & 0 & 89 & 88 & 205 & 39 \\
\hline sctap3 & 0.55 & 2 & 3.00 & 0 & 135 & 277 & 135 & 0 & 42 & 235 & 277 & 425 \\
\hline pilotwe & 1.94 & $3 / 6$ & 7.53 & 114 & 106 & 1 & 208 & 12 & 1 & 0 & 85 & 100 \\
\hline maros & 0.42 & 2 & 1.58 & 6 & 114 & 14 & 120 & 0 & 2 & 12 & 18 & 241 \\
\hline fit1p & 0.32 & 1 & 0.03 & 0 & 0 & 0 & 0 & 0 & 0 & 0 & 0 & 1 \\
\hline & 0.66 & 2 & 1.82 & 3 & 44 & 18 & 47 & 0 & 6 & 12 & 26 & 67 \\
\hline & 0.61 & 2 & 0.77 & 0 & 33 & 60 & 33 & 0 & 17 & 43 & 93 & 34 \\
\hline ship08l & 0.67 & 2 & 0.83 & 0 & 44 & 0 & 44 & 0 & 0 & 0 & 0 & 91 \\
\hline pilotnov & 0.71 & $3 / 1$ & 11.77 & $\overline{94}$ & 0 & 1194 & 94 & $\overline{0}$ & 462 & 748 & 288 & 5 \\
\hline nest & 0.74 & $4 / 1$ & 8.93 & 64 & 1209 & 228 & 186 & 1087 & 106 & 122 & 498 & 96 \\
\hline fit1d & 0.23 & 1 & 0. & 0 & 0 & 0 & 0 & 0 & 0 & 0 & 0 & 0 \\
\hline bnl & 0.97 & & 5.85 & 0 & 57 & 502 & 57 & 0 & 168 & 334 & 519 & 297 \\
\hline pilotja & 1.40 & & 6.30 & 47 & 65 & 99 & 109 & 3 & 41 & 58 & 128 & 49 \\
\hline ship12l & 0.89 & & 1.38 & 0 & 61 & 1 & 61 & 0 & 0 & 1 & 1 & 223 \\
\hline & 0.70 & 2 & 5.20 & 0 & 108 & 410 & 108 & 0 & 157 & 253 & 410 & 570 \\
\hline$a u 3 b$ & 2.03 & 2 & 10.15 & 10 & 380 & 94 & 386 & 4 & 48 & 46 & 161 & 650 \\
\hline degen3 & 1.03 & $2 / 1$ & 34.92 & 0 & 918 & 2 & 918 & 0 & 1 & 1 & 3 & 628 \\
\hline greenbea & 2.46 & $2 / 4$ & 17.72 & 70 & 376 & 235 & 442 & 4 & 61 & 174 & 289 & 992 \\
\hline
\end{tabular}

Table V: Basis recovery performance (cont.) 


\begin{tabular}{|c|c|c|c|c|c|c|c|c|c|c|c|c|}
\hline \multirow[b]{3}{*}{ name } & \multicolumn{2}{|c|}{ setup } & \multicolumn{4}{|c|}{ solve } & \multicolumn{4}{|c|}{ pivot type } & \multirow{2}{*}{\multicolumn{2}{|c|}{$\frac{\text { Megiddo }}{\text { pivots }}$}} \\
\hline & \multirow[b]{2}{*}{ secs } & \multirow[b]{2}{*}{ fact } & \multirow[b]{2}{*}{ secs } & \multicolumn{3}{|c|}{ pivots } & \multicolumn{2}{|c|}{ simplex } & \multicolumn{2}{|c|}{\begin{tabular}{|l|} 
cleanup \\
\end{tabular}} & & \\
\hline & & & & ph. 1 & ph. 2 & cleanup & exch. & push & exch. & push & primal & dual \\
\hline greenbeb & $\overline{2.82}$ & $3 / 4$ & 16.75 & 58 & 301 & 220 & 348 & 11 & 69 & 151 & 324 & 966 \\
\hline $\mathrm{d} 2 \mathrm{q} 06 \mathrm{c}$ & 2.27 & 2 & 10.53 & 2 & 139 & 31 & 139 & 2 & 13 & 18 & 43 & 252 \\
\hline woodw & 1.39 & 2 & 4.88 & 121 & 114 & 320 & 191 & 44 & 0 & 320 & 401 & 129 \\
\hline pilo & 13.60 & 4 & 17.57 & 8 & 49 & 54 & 49 & 8 & 18 & 36 & 86 & 39 \\
\hline fit2] & 3.47 & 3 & 3.05 & 4 & 5 & 0 & 9 & 0 & 0 & 0 & 4 & 9 \\
\hline stocfor3 & 6.45 & 2 & 330.25 & 197 & 2075 & 0 & 2272 & 0 & 0 & 0 & 364 & 3191 \\
\hline wood1p & 1.13 & 2 & 3.40 & 57 & 112 & 0 & 165 & 0 & 0 & 0 & 0 & 54 \\
\hline & 48.44 & 3 & 45.58 & 6 & 42 & 26 & 45 & 3 & 5 & 21 & 42 & 22 \\
\hline fit $2 d$ & 2.90 & 2 & 1.78 & 1 & 6 & 3 & 7 & 0 & 1 & 2 & 6 & 2 \\
\hline total & 114.38 & & $\overline{591.43}$ & & & & & & & & & \\
\hline
\end{tabular}

Table VI: Basis recovery performance (cont.) 